\title{
Monitor placement, sources of variance and reliability of free-living physical activity: a pilot investigation
}

\author{
Ian Cook (BA (Phys Ed) Hons, BSc (Med) Hons) ${ }^{1}$ \\ Estelle V Lambert (PhD) ${ }^{2}$ \\ ${ }^{1}$ Physical Activity Epidemiology Laboratory, University of Limpopo (Turfloop Campus), South Africa \\ ${ }^{2}$ MRC/UCT Research Unit for Exercise Science and Sports Medicine, University of Cape Town Medical School, South Africa
}

\begin{abstract}
Objectives. We investigated the effect of monitor placement on the sources of variance and reliability of objectively measured free-living physical activity (PA).

Design. A convenience sample ( $N=7 ; 3$ females, 4 males) of rural, adult, black South Africans was recruited from the plantation section of a local lumber mill. PA was assessed using two uni-axial accelerometers placed on the right hip $(\mathrm{RH})$ and left hip $(\mathrm{LH})$, over 3 weekdays. PA indices were total counts, average counts, inactivity $\left(0-499\right.$ counts. $\left.\mathrm{min}^{-1}\right)$, moderate- 1 activity (500 - 1951 counts. min $^{-1}$ ) and moderate-2+vigorous activity ( $\geq 1952$ counts. $\min ^{-1}$ ).
\end{abstract}

Results. Accelerometer output did not differ across trials for either hip placement $(p>0.2)$. There were no significant differences between $\mathrm{RH}$-LH for any accelerometer variable $(p>0.1)$ and effect sizes were small $(0.02-0.15)$. Monitor position did not contribute any variance to accelerometer variables. Variance due to monitor unit contributed $<2 \%$ to raw and derived accelerometer variables. Coefficients of variation derived from the standard deviation of $\mathrm{RH}-\mathrm{LH}$ transformed differences ranged from $2.7 \%$ to $10.5 \%$, except for moderate-1 and moderate-2+vigorous variables (16.0 - 72.8\%). Intraclass correlation coefficients (ICC, RH-LH) were $>0.90$, except for moderate-1 time accumulated in bouts $\geq 10$ $\min (\mathrm{ICC}=0.83)$. Agreement between RH-LH for achieving CDC/ ACSM PA guidelines was significant (kappa $=0.79, p=0.002$ )

Conclusions. Firstly there was no statistically significant difference between RH-LH for any accelerometer variable. Secondly, accelerometer units accounted for little of the variance in accelerometer variables. Thirdly, greater variability in monitor placement was apparent for moderate- 1 and moderate-2+vigorous variables.

\section{CORRESPONDENCE:}

Ian Cook

Physical Activity Epidemiology Laboratory

University of Limpopo (Turfloop Campus)

PO Box 459

Fauna Park,

0787 Polokwane, South Africa

Tel+fax: +27 152682390

E-mail: ianc@ul.ac.za

\section{Introduction}

The use of accelerometers by researchers to objectively monitor physical activity has seen a dramatic increase since $1997 .{ }^{1}$ With the greater use of uni-axial accelerometers in large field studies, ${ }^{2}$ important questions arise relating to possible sources of measurement error, such as monitor placement. ${ }^{3,4}$ To date a number of laboratorybased studies have considered the effects of different placement positions on the output of uni-axial accelerometers ${ }^{5,6}$ and the intra- and inter-instrument variability of uni-axial accelerometers using mechanical settings ${ }^{7-9}$ or motorised treadmill trials. ${ }^{7,10-13}$ Few studies have employed free-living protocols when evaluating the effect of placement position of movement monitors or the inter-instrument reliability of movement monitors. $5,14,15$

Only one free-living study has considered the placement position or inter-instrument reliability of uni-axial accelerometers by having subjects wear two monitors on either side of the hip. ${ }^{14}$ That study was carried out in a highly urbanised setting, during the waking hours of one highly structured day, using students and staff from a university setting who were recreational runners and accumulated significant amounts of vigorous physical activity. ${ }^{14}$ Moreover, in their statistical analyses, McClain et al. ${ }^{14}$ did not report the possible effect of monitor placement on variance distribution ${ }^{16}$ nor did they examine agreement ${ }^{17}$ between the two placement positions. Consequently, similar and more expanded analyses of free-living samples with more variable day-to-day physical activity patterns are required. Importantly, rural subjects with low, recreational (vigorous) physical activity but high work-related (moderate) physical activity demands should be recruited.

Uni-axial accelerometers demonstrate low intra-unit variability but do exhibit inter-unit variability such that post-measurement adjustment in multivariate analysis has been used to account for this variance which could otherwise dilute the true relationship between a health outcome and accelerometer output. ${ }^{18}$ In this regard, Welk et al. showed that $0.9 \%$ of the variance for the raw counts obtained during multi-speed treadmill trials could be attributed to individual uni-axial accelerometer units. ${ }^{6}$ There is thus a need to determine if this variance due to inter-unit variability is present or possibly even greater in free-living conditions than has been observed in laboratory trials.

The objectives of this pilot investigation were firstly to evaluate the effect of monitor placement and monitor units on the variance distribution in relation to other sources of variance, and secondly to determine if monitor position had practically significant effects on reliability statistics, specifically in an adult population exhibiting low levels of vigorous, recreational physical activity but high levels of moderate work-related physical activity. 


\section{Methods}

\section{Study protocol}

The accelerometry data used in this analysis were collected during the validity trial of the International Physical Activity Questionnaire (IPAQ). ${ }^{19-21}$ Briefly, participants were recruited to wear two uni-axial accelerometers and contacted twice over an 8-day period. On the first occasion, subjects were recruited, provided anthropometric data and were instructed on the necessary procedures for wearing the accelerometers, one on either side of the hip $(\mathrm{RH}=$ right-hand side hip, $\mathrm{LH}=$ left-hand side hip). Eight days later the accelerometers were collected. Subjects received a small honorarium on completion of the study. Signed informed consent was obtained from all participants. The study was approved by the Ethics Committee of the University of Limpopo (Turfloop Campus).

\section{Subjects}

A convenience sample of 7 black adult males $(N=4,30.0 \pm 0.8$ yrs, $175.7 \pm 3.2 \mathrm{~cm}, 63.8 \pm 5.9 \mathrm{~kg})$ and females $(N=3,38.7 \pm 7.0 \mathrm{yrs}$, $155.5 \pm 4.8 \mathrm{~cm}, 56.4 \pm 4.5 \mathrm{~kg}$ ), resident on farms and villages, were recruited from the plantation section of a local lumber mill situated in rural Limpopo Province, South Africa. All participants recruited were $\mathrm{BMI}<27 \mathrm{~kg} \cdot \mathrm{m}^{-2}$. These forestry workers performed a variety of manual tasks and ensured that plantations were created and maintained, and that raw timber was harvested, sized, cleaned and stacked prior to transport to the saw mill for further processing.

\section{Physical activity counts and durations}

To objectively quantify free-living physical activity of the subjects, two uni-axial accelerometers were worn for at least 8 days. The CSA model 7164 (Computer Science Applications, Inc. Shalimar, FL), now marketed as the MTI Actigraph (MTI Health Services, Fort Walton Beach, $\mathrm{FL})$, is small and unobtrusive $(5.1 \mathrm{~cm} \times 4.1 \mathrm{~cm} \times 1.5$ $\mathrm{cm}, 42.6 \mathrm{~g}) .{ }^{22}$ The accelerometers were worn on the $\mathrm{RH}$ and $\mathrm{LH}$, securely attached to a nylon belt. The accelerometers could be removed for sleeping and bathing purposes by unclipping the nylon belt. Subjects were carefully instructed as to the proper positioning of the accelerometers (mid-axillary line). The minute-by-minute data were downloaded from the accelerometers onto an IBM-compatible personal computer via an interface unit, for further analysis using proprietary software (DAYBYDAY.XLS, Microsoft Excel 97 macro) and a customised data reduction programme (Microsoft Excel 2002 macro). Physical activity counts were defined as total counts (counts. day $^{-1}$ ) and average counts (counts.day ${ }^{-1} \cdot \mathrm{min}^{-1}=$ total counts/registered time for counts. $\mathrm{min}^{-1} \geq 1$ ). Physical activity volumes (min.day ${ }^{-1}$ ) of inactivity and moderate and vigorous activity were derived using previously defined cut-points. ${ }^{14,16}$ Inactivity (lying, sitting, standing quietly, light activity) was classified as $0-499$ counts. min $^{-1}$. For moderate activity $(3-6 \mathrm{METs}, 1 \mathrm{MET}=1$ metabolic equivalent $=3.5$ $\mathrm{mlO}_{2} \cdot \mathrm{kg}^{-1} \cdot \mathrm{min}^{-1}=1 \mathrm{kcal} \cdot \mathrm{kg}^{-1} \cdot \mathrm{hr}^{-1}$ ) a distinction was made between activities requiring less ambulation (moderate-1: house work, yard work) and predominantly ambulatory activities (moderate-2: walking). The cut-points for moderate- 1 and moderate- 2 were defined as $500-1591$ counts.min-1 and $1592-5724$ counts. min $^{-1}$, respec-

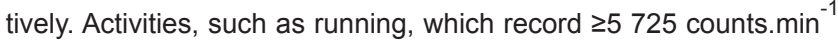
were defined as vigorous ( $>6 \mathrm{METs}$ ). The amount of activity accumulated in bouts of $\geq 10 \mathrm{~min}$ (bouts separated by at least $1 \mathrm{~min}$ ) for the moderate- 1 and moderate-2+vigorous categories were also derived. The first and last days of the 8-day monitoring period were excluded. Only weekdays with at least 8 hours.day ${ }^{-1}\left(480\right.$ minutes.day $\left.{ }^{-1}\right)$ of registration (counts.min-1 $\geq 1$ ) were considered. This would repre- sent the minimum of a 40-hour, 5-day working week in this sample. Valid accelerometer data for the first 3 weekdays were used for all subsequent analyses. A 3-weekday period was selected because 3 subjects provided a minimum of 3 weekdays of valid accelerometer data.

\section{Statistical analysis}

Descriptive statistics comprised means and standard deviations. If data distribution was non-normal, the parametric results were confirmed with transformed (natural logarithms) or ranked data. Data were analysed using appropriate statistical software (SPSS for Windows 13.0). Confidence intervals $(95 \% \mathrm{Cl})$ were calculated as required. Significance for all inferential statistics was set at $p<0.05$.

To evaluate the sources of variability in accelerometer data, variance components in mixed effects models were estimated using restricted maximum likelihood methods. ${ }^{16}$ Accelerometer raw output and derived indices were the dependant variables for these analyses. Variance components were estimated for subject (interindividual) variance, monitor unit variance ( 8 units), trial variance (3 trials), monitor position variance ( 2 positions), and residual (intraindividual) variance. The variance components were expressed as a percentage of the total variance. Inter-individual variance represents true variation between subjects while intra-individual variance represents day-to-day variation within subjects. The variance due to monitor unit, trial and monitor position effects were nested within subjects. Gender and day of the week were entered as fixed factors. Using the same set of fixed and random factors, a separate analysis was performed on the raw accelerometer data (counts.day ${ }^{-1}$ ) to test for possible interaction of subjects by monitors. ${ }^{11}$

A multivariate, two-way repeated-measures ANOVA was used to examine possible differences across trials for $\mathrm{RH}$ and $\mathrm{LH}$ accelerometer variables. Separate analyses were conducted with gender entered as a between-subjects factor, and interactions between gender and hip placement or trial. In order to compare the accelerometer output between $\mathrm{RH}$ and $\mathrm{LH}$, the mean difference and limits of agreement for repeated measurements were calculated for all accelerometer variables. ${ }^{17}$ We did not use the $\mathrm{RH}$ and LH withinsubject variances across trials to adjust the variance of differences. Within-subject variances for any accelerometer variable did not differ significantly between $\mathrm{RH}$ and $\mathrm{LH}(p>0.4)$ such that any adjustment using within-subject variance would simply inflate the variance of differences. This inflation would simply mean that within-subject variance across trials was greater than the $\mathrm{RH}$-LH difference variance. Bland-Altman plots and $\mathrm{Q}-\mathrm{Q}$ normal probability plots of difference scores were constructed to visually assess the limits of agreement and distribution of difference scores, respectively. ${ }^{17}$ For comparative purposes $^{14}$ we also calculated a coefficient of variation (CV) from the standard deviation of the differences of the transformed raw data (natural logarithms) using the following formula $\left(\mathrm{CV}=100 \times\left[e^{\mathrm{SD}}-1\right]\right){ }^{23}$ Because the moderate-2+vigorous (bouts $\geq 10 \mathrm{~min}$ ) contained zero values, we added one to all raw values before taking the natural logarithms of the raw values. Effect sizes $(d)$ were calculated from the mean difference and the standard deviation of the differences obtained during the limits of agreement analysis (raw values), and interpreted according to Cohen's conventions; 0.20 (small), 0.50 (moderate), 0.80 (large). ${ }^{24}$ The $I C_{C}$ (intraclass correlation coefficient; average measure, absolute agreement, two-way random effects) ${ }^{25}$ was calculated (RH v. LH) for each trial. Thereafter, the mean $\mathrm{ICC}_{\mathrm{m}}$ was calculated from the $\mathrm{ICC}_{\mathrm{m}}$ of three trials.

Subjects were also classified according to the ACSM/CDC guidelines ( $\geq 30$ min $^{\text {day }}{ }^{-1}$ of moderate-2+vigorous activity accumu- 
lated in bouts $\geq 10 \mathrm{~min})^{26}$ for each trial and monitor position. Agreement between $\mathrm{RH}$ and $\mathrm{LH}$ for each trial (7 pairs of data) and for all trials combined ( 21 pairs of data) was assessed by constructing 2-by-2 tables and calculating Cohen's kappa ( $\kappa)$ statistic.

\section{Results}

Six of the 8 units were used twice; once on the $\mathrm{RH}$ and once on the $\mathrm{LH}$. Registered time for counts $\geq 1$ ranged from $8.12 \mathrm{hrs}$ to $14.45 \mathrm{hrs}$ (10.18 $\pm 1.75 \mathrm{hrs}$ ) and did not differ significantly between $\mathrm{RH}$ and $\mathrm{LH}$ monitors (RH: $10.18 \pm 1.91 \mathrm{hrs}$; LH: $10.18 \pm 1.63 \mathrm{hrs;} \mathrm{mean} \mathrm{difference}$ $=-0.01 \mathrm{hrs}, p=0.9771)$. There was no significant difference across trials for registered time for $\mathrm{RH}$ or LH monitors $(p>0.2)$.

Descriptive statistics for $\mathrm{RH}$ and $\mathrm{LH}$ accelerometer raw output and derived variables are reported in Table I. Because the volume of vigorous activity accumulated was low (RH: $6 \pm 12$ min.day $^{-1}$, LH: $6 \pm 11$ min. day $^{-1}$ ) and distribution was skewed, a separate variable was constructed which combined moderate- 2 and vigorous activity. Repeated-measures analyses found no significant differences across the three trials for any accelerometer variable $(p>0.1)$ nor any significant differences between hip placement or gender for any accelerometer variable $(p>0.4)$, except for moderate- 1 (bouts $\geq 10$ min) where females accumulated significantly more activity than males (34 min, $p=0.0081$ ) (Table I). There was no interaction between gender and trial or hip placement $(p>0.2)$. There was no marked difference in significance using the raw data or rank transformed data for the moderate-2+vigorous (bouts $\geq 10 \mathrm{~min}$ ) variable, consequently the $p$-values from the raw data are reported.

Effect sizes for the mean differences between $\mathrm{RH}$ and $\mathrm{LH}$ ranged from $d=0.02$ to $d=0.15$ (small effect) for all accelerometer variables. The 95\% limits of agreement in Table I represent the area within which $95 \%$ of the mean differences would be expected to fall, if the differences are normally distributed. ${ }^{17}$ The difference scores (RH minus $\mathrm{LH}$ ) were randomly distributed irrespective of the magnitude of the accelerometer variables and the difference scores were normally distributed (data not shown). Agreement analysis revealed no systematic bias in mean differences for any accelerometer variables. Limits of agreement were similar for moderate- 1 and moderate-2+vigorous variables (Table I).

The distribution of variance is reported in Table II. Monitor position did not contribute to the variability of any of the accelerometer variables. The variability due to individual monitor units was $0.3 \%$ to $1.7 \%$ for raw and derived accelerometer data. There was no interaction for subjects-by-monitors. Inter-individual variance was

\section{Table I. Descriptive statistics for accelerometer raw output and derived indices}

\begin{tabular}{|c|c|c|c|c|}
\hline \multirow{2}{*}{$\begin{array}{l}\text { Raw output and derived } \\
\text { indices }\end{array}$} & \multicolumn{3}{|c|}{ Monitor positions } & \multirow[t]{2}{*}{ Agreement } \\
\hline & Right-hand side hip ${ }^{* *}$ & Left-hand side hip ${ }^{* *}$ & Significance ${ }^{\dagger \dagger}$ & \\
\hline Total counts * & 761635 (212 074) & 763290 (178 772) & 0.2755 & $-1655(-151016$ to 147706$)$ \\
\hline Average count $^{\dagger}$ & $999(215)$ & 1008 (177) & 0.2376 & $-9(-140$ to 122$)$ \\
\hline Inactivity $\ddagger \S$ & $1026(102)$ & $1024(91)$ & 0.6197 & $+2(-50$ to 54$)$ \\
\hline \multicolumn{5}{|l|}{ Moderate-1 $\neq \|$} \\
\hline (bouts $\geq 1 \mathrm{~min}$ ) & $284(62)$ & 285 (59) & 0.1305 & $-1(-33$ to 30$)$ \\
\hline (bouts $\geq 10 \mathrm{~min}$ ) & $44(23)$ & $46(19)$ & 0.1854 & $-2(-24$ to 20$)$ \\
\hline \multicolumn{5}{|l|}{ Moderate- 2 + vigorous $\ddagger \mathbb{\pi}$} \\
\hline (bouts $\geq 1 \mathrm{~min}$ ) & $130(50)$ & $131(40)$ & 0.1529 & $0(-36$ to 35$)$ \\
\hline (bouts $\geq 10 \mathrm{~min}$ ) & $19(22)$ & $20(18)$ & 0.9841 & $-1(-20$ to 19$)$ \\
\hline
\end{tabular}

\section{Table II. Variance component analysis of 3-day accelerometry raw output and derived variables}

\begin{tabular}{|c|c|c|c|c|c|c|c|}
\hline \multirow[b]{3}{*}{ Sources of variance ${ }^{\dagger}$} & & & \multirow[b]{3}{*}{ Inactivity } & \multicolumn{4}{|c|}{ Activity duration * } \\
\hline & \multicolumn{2}{|c|}{ Activity counts * } & & \multicolumn{2}{|c|}{ Moderate-1 } & \multicolumn{2}{|c|}{ Moderate-2 + Vigorous } \\
\hline & Total & Average & & Bouts $\geq 1 \mathrm{~min}$ & Bouts $\geq 10 \mathrm{~min}$ & Bouts $\geq 1 \mathrm{~min}$ & Bouts $\geq 10 \mathrm{~min}$ \\
\hline Inter-individual & 79.0 & 67.1 & 68.4 & 60.7 & 23.5 & 68.8 & 53.8 \\
\hline Intra-individual & 21.0 & 32.9 & 31.6 & 39.3 & 76.5 & 31.2 & 46.2 \\
\hline Position $¥$ & 0.0 & 0.0 & 0.0 & 0 & 0.0 & 0.0 & 0.0 \\
\hline Trial $^{\S}$ & 3.1 & 7.3 & 23.7 & 29.1 & 10.1 & 8.2 & 0.0 \\
\hline Monitor 1 & 1.7 & 0.0 & 0.7 & 0 & 0.3 & 0.0 & 0.0 \\
\hline Residual & 16.2 & 25.6 & 7.2 & 10.2 & 66.1 & 23.0 & 46.2 \\
\hline Total & 100 & 100 & 100 & 100 & & 100 & 100 \\
\hline
\end{tabular}


Table III. Reliability analysis of 3-day accelerometry raw output and derived indices

\begin{tabular}{|c|c|c|c|c|c|}
\hline \multirow[b]{2}{*}{ Raw output and derived indices * } & \multirow[b]{2}{*}{ Coefficient of variation $^{\dagger}$} & \multicolumn{4}{|c|}{ Intraclass correlation coefficient $^{\ddagger}$} \\
\hline & & Trial 1 & Trial 2 & Trial 3 & Mean" \\
\hline Total counts & 10.5 & 0.95 & 0.97 & 0.96 & 0.96 \\
\hline Average counts & 6.3 & 0.98 & 0.92 & 0.96 & 0.95 \\
\hline Inactivity & 2.7 & 0.93 & 0.98 & 0.94 & 0.95 \\
\hline \multicolumn{6}{|l|}{ Moderate-1 } \\
\hline (bouts $\geq 1 \mathrm{~min}$ ) & 5.7 & 0.86 & 0.99 & 0.98 & 0.94 \\
\hline (bouts $\geq 10 \mathrm{~min}$ ) & 33.8 & $0.72^{\S}$ & 0.78 & $0.39 \S$ & 0.63 \\
\hline \multicolumn{6}{|l|}{ Moderate-2 + vigorous } \\
\hline (bouts $\geq 1 \mathrm{~min}$ ) & 16.0 & 0.96 & 0.89 & 0.96 & 0.94 \\
\hline (bouts $\geq 10 \mathrm{~min}$ ) & 72.8 & $0.61 \S$ & 0.92 & 0.95 & 0.83 \\
\hline
\end{tabular}

greater than intra-individual variance for all accelerometer variables, except the moderate-1 (bouts $\geq 10 \mathrm{~min}$ ) variable. Of all the variance components nested within-subjects, trial contributed the most. There was no marked difference in variance components using the raw data or rank transformed data for the moderate-2+vigorous (bouts $\geq 10 \mathrm{~min}$ ) variable, consequently the variance from the raw data is reported in Table II. The variance components reported in Table II for the moderate-1 (bouts $\geq 10 \mathrm{~min}$ ) variable was obtained from natural logarithm transformation.

The reliability analysis is reported in Table III. CV ranged from $2.7 \%$ to $10.5 \%$ for raw and derived variables, except for moderate- 1 and moderate-2+vigorous variables ( $16.0 \%$ to $72.8 \%)$. ICCm for four of the six accelerometer variables were $>0.90$. The $I C C_{m}$ for trial 1 and trial 3 of the moderate- 1 (bouts $\geq 10 \mathrm{~min}$ ) variables were low and insignificant, resulting in a low mean $I C C_{m}$. Transforming the moderate-1 (bouts $\geq 10 \mathrm{~min}$ ) data did not improve the results. The high intra- to inter-subject variance ratio for the moderate-1 (bouts $\geq 10 \mathrm{~min}$ ) data (Table II) would account for the continuing low ICCm. In contrast, the low $\mathrm{ICC}_{\mathrm{m}}$ for the moderate-2+vigorous (bouts $\geq 10$ $\mathrm{min}$ ) reported in Table III was the result of averaging an insignificant $I C C m$ for trial 1 and significant ICC $m$ for trials 2 - 3. Rank transforming the moderate-2+vigorous (bouts $\geq 10 \mathrm{~min}$ ) data resulted in significant reliability coefficients (consistency, two-way random effects) for all trials $(p \leq 0.01)$ resulting in a mean $I C C_{m}=0.92 .{ }^{25}$

Only two subjects were misclassified in terms of achievement of ACSM/CDC guidelines between RH and LH monitors; one subject on trial 1 and one subject on trial 3 . There were no misclassifications for trial 2. Agreement between hip positions was significant for trial $2(\kappa=1.00, p=0.0082)$ but not trials 1 and $3(\kappa=0.70, p=0.05334)$. Overall (trial 1-3) agreement was significant $(\kappa=0.79, p=0.0002)$.

\section{Discussion}

This study is the first analysis, albeit preliminary in nature, which has reported on the effect of monitor placement on the variance distribution and reliability of uni-axial accelerometer output and derived variables obtained during free-living conditions in a South African setting. The principal findings of this analysis were firstly that there were no statistically significant differences between hip positions for raw or derived accelerometer variables. Secondly, greater variability was apparent for the moderate-2+vigorous variables, especially so for time accumulated in bouts $\geq 10 \mathrm{~min}$ for both moderate categories. Thirdly, individual accelerometer units accounted for nearly $2 \%$ of the variance for raw accelerometer counts.
Welk et al. were the first to demonstrate that at a moderate treadmill walking speed of $4.8 \mathrm{~km} \cdot \mathrm{hr}^{-1}$, different uni-axial accelerometer hip positions (anterior axillary, mid-axillary, posterior axillary) had a significant effect $(p<0.05)$ on accelerometer output $\left(\right.$ cts. min $\left.^{-1}\right)$ such that variability (percentage of mean score) was $30 \%$ compared with $3 \%$ for two other accelerometers (Biotrainer and Tritrac). ${ }^{6}$ These findings suggest that variability is more likely to increase at moderate intensities during free-living trials where monitor position is not accurately controlled.

Our results are in agreement with laboratory treadmill studies that have found greater variability between uni-axial accelerometers mounted across hips ( $\mathrm{RH}$ v. $\mathrm{LH}$ ) at moderate intensities compared with vigorous intensities. ${ }^{7,10}$ Nichols et al. found lower reliability across hip placement for slow speeds $\left(\right.$ ICC $\left.=0.55,3.2 \mathrm{~km} . \mathrm{hr}^{-1}\right)$ compared with faster speeds $\left(I C C=0.91,6.4 \mathrm{~km} . \mathrm{hr}^{-1}\right) .7$ Similarly, Brage et al. demonstrated lower agreement at moderate walking speeds $\left(4-6 \mathrm{~km} \cdot \mathrm{hr}^{-1}\right)$ compared with faster running speeds $(8-14$ $\mathrm{km} \cdot \mathrm{hr}^{-1}$ ), even though ICC $>0.911^{10}$ Importantly, these differences remained even after calibration of the individual monitors.

The effect of hip v. lower back positions on uni-accelerometer output has been investigated during laboratory treadmill ${ }^{5}$ and freeliving trials. ${ }^{5,15}$ Yngve et al. reported significant differences $(p<0.01)$ between hip and lower back positions for normal and fast walking and jogging $\left(4.3,5.8\right.$ and $9.6 \mathrm{~km} . \mathrm{hr}^{-1}$, respectively) irrespective of setting (indoor athletics track or treadmill). ${ }^{5}$ The absolute percentage error (|[Hip - Back/Back]| $\times 100$ ) was greatest for the normal to fast walking (9.5\% and $7.2 \%$, respectively) which occurred in the moderate (1 $\left.952-5724 \mathrm{cts} . \mathrm{min}^{-1}\right)$ range, and lowest for jogging $\left(4.5 \%\right.$; $\geq 5725 \mathrm{cts}$. $\mathrm{min}^{-1}$ range). It was suggested that the differential findings between walking and jogging were due to changes in vertical displacement of the hip compared with the lower back during the transition from fast walking to jogging. ${ }^{5}$ During a 7 -day free-living trial, no significant difference was found between monitor positions (10 cts. min $^{-1}, p=0.23,95 \%$ limits of agreement: -102 to 82 cts. min $^{-1}$ ). Moreover, the amount of daily moderate-to-vigorous time predicted from four METs v. cts. min $^{-1}$ equations did not differ between monitor positions. ${ }^{5}$ During a 4-day free-living trial in children, Nilsson et al. demonstrated no difference between hip and lower back positions for raw counts $\left(22 \mathrm{cts} \cdot \mathrm{min}^{-1}, p=0.20,95 \%\right.$ limits of agreement: -110 to $\left.154 \mathrm{cts} \cdot \mathrm{min}^{-1}\right) .{ }^{15}$ The wide limits of agreement was due to 2 subjects and might have been caused by specific movements that result in differences in movements between the hip and lower back. A significant difference $(p<0.01)$ between output for hip and lower back positions was found only when data were sampled over short periods ( 5 sec epochs) and only in the moderate range (1 952 - 5725 
cts. $\left.\min ^{-1}\right)$. Short sampling periods might be more likely to capture even small differences in movement at different body sites. ${ }^{15}$

More recently, McClain et al. have been the first to show that in free-living conditions, $\mathrm{RH}-\mathrm{LH}$ comparisons exhibit greater variability for the moderate- 2 range $\left(500-1951 \mathrm{cts} \cdot \mathrm{min}^{-1}\right),{ }^{14}$ thus confirming the laboratory treadmill findings of greater variability at walking speed intensities. ${ }^{7,10}$ Our results are in accord with the lower variability reported by $\mathrm{McClain}$ et al. for total counts, inactivity and moderate-1 variables. ${ }^{14}$ However, in contrast to McClain et al., we have found higher variability for the moderate-2+vigorous physical activity (MVPA) categories ( $\geq 1952 \mathrm{cts}^{-\mathrm{min}^{-1}}$; bouts $\geq 1 \mathrm{~min}$ and $\geq 10 \mathrm{~min}){ }^{14}$ These apparently contradictory findings are due to the selective subject recruitment strategies employed in this study and by McClain et al. ${ }^{14}$

McClain et al. purposively selected highly active individuals (runners), which resulted in approximately $65 \%$ of MVPA time being accumulated from vigorous activity ( $\geq 5725$ cts.min-1). ${ }^{14}$ Consequently, the increased variability of the moderate- 2 range was diluted by the larger contribution of the vigorous range. The present study, on the recommendation of McClain et al., ${ }^{14}$ selected subjects who were not vigorously active but rather accumulated activity in the moderate- 1 and moderate- 2 activity zones. This is evident by a 3-fold greater moderate-1 time, a nearly 5 -fold greater moderate2 time and an 8-fold lower vigorous time, compared with McClain et al. ${ }^{14}$ Moreover, the moderate- 2 zone in this study contributed approximately $95 \%$ to the MVPA variable. Consequently, in contrast to McClain et al., ${ }^{14}$ we do not recommend collapsing moderate-2 and vigorous activity zones into a single MVPA variable as a method to dilute greater variability from the moderate- 2 zone. We would caution researchers that the creation of an MVPA variable does not always imply a reduced variability as suggested by others. ${ }^{14}$

Taken together these studies have shown that uni-axial accelerometer counts sampled during moderate intensities are more variable when collected at different body sites. $5-7,10,14,15$ However, none of these studies investigated the effect of body sites on moderate-to-vigorous time accumulated in bouts $\geq 10 \mathrm{~min}$ and on the compliance of subjects with PA public health guidelines. Consequently, contrary to the assertion by Trost et al. that the practical effect of different body sites on uni-axial accelerometer output is negligible, ${ }^{3}$ we would argue that there is still uncertainty as to the practical effects of mounting uni-axial accelerometers at different body sites.

Although we could not calibrate the units before and during the field measurements, post-measurement variance analysis showed that $1.7 \%$ of the variability for total daily counts could be attributed to monitor units, which is in agreement with the $0.9 \%$ found by Welk et al. during multi-speed treadmill trials. ${ }^{11}$ The twofold increase in variability compared with Welk et al. might be due to the greater time spent at low and moderate intensities in this sample and the freeliving conditions. Importantly, the $14.5-20.1 \%$ variance for the trialby-subject and monitor-by-trial-by-subject interaction reported by Welk et al. suggests differences by subject due to clothing, posture and other anthropometric variations, which could be amplified during free-living trials due to lack of standardisation. ${ }^{11}$ Moreover, Brage and associates have shown greater inter-unit variability at low to moderate intensities ${ }^{9,10}$ and it has been suggested that real anatomical differences such as body size, hip geometry and hip softtissue deposition might explain acceleration differences between hip positions at lower movement intensities, specifically slow to normal walking. $^{10}$
We reanalysed uni-axial accelerometer data from a recently reported variance component analysis ${ }^{27}$ and found that accelerometer units accounted for $0 \%$ and $5.8 \%$ of the total variance of daily counts in rural and urban subjects, respectively. It is possible that the greater time spent in low-to-moderate activities (low accelerations) by the urban subjects would result in greater inter-instrument variability. ${ }^{9,10}$ This could in turn account for the increased accelerometer unit variability in urban subjects. Our results and those of others ${ }^{9-11}$ highlight the need to make post-measurement statistical adjustments for monitor units if individual calibration of units cannot be performed before and during field trials. $9,10,18$

Our finding of a substantial within-subject variance for the moderate-1 (bouts $\geq 10 \mathrm{~min}$ ) in comparison with other variables is difficult to explain. We reanalysed uni-axial accelerometer data recently reported for a larger rural sample out of which the current sample was drawn. ${ }^{27}$ As with the current analysis, the between- and within-subject variance was $30 \%$ and $70 \%$, respectively. Further analyses are required to determine whether the variance distribution for this particular accumulated uni-axial accelerometer variable is due to a bias resulting from the creation of a variable accumulated from bouts of $P A \geq 10 \mathrm{~min}$ or is a reflection of a true behavioural characteristic of the subjects.

The strengths of the present pilot study are firstly the uniqueness of the analysis within a South African context. Secondly, this analysis provides reliability and variance estimates for a South African sample with particularly high occupational physical activity demands. Thirdly, this study has also been the first to show dramatically increased variability for time in moderate intensity variables accumulated in bouts $\geq 10 \mathrm{~min}$. Further research is required to confirm this increased variability, and possible causes for this phenomenon. The weakness of this study is firstly that we could not differentiate between mechanical or anatomical causes for the increased variability of uni-axial accelerometer output at low to moderate intensities. However, no study to date has been able to quantify the separate contributions of mechanical and anatomical causes to increased variability across hip placement. Secondly, our sample size was limited when investigating the effects of monitor position variability on the compliance with public health PA guidelines.

In conclusion, this analysis has demonstrated that uni-axial accelerometer placement across hips has a greater effect on variability for derived duration variables in the moderate intensity range. This increased variability resulted in statistically small effects, and future research should consider investigating the effects of uni-axial accelerometer placement on bouts of activity and the consequent compliance with PA guidelines. As part of quality control procedures, researchers should perform similar analyses when conducting field trials, and should either regularly calibrate individual accelerometer units or adjust statistically post-measurement when relating accelerometer output to a health outcome.

\section{Acknowledgements}

The Research Development and Administration Division of the University of Limpopo (Turfloop Campus) and the Research Capacity Development Group of the Medical Research Council of South Africa supported this study.

\section{REFERENCES}

1. Troiano RP. A timely meeting: objective measurement of physical activity. Med Sci Sports Exerc 2005;37:S487-S489.

2. Troiano RP. Large-scale applications of accelerometers: new frontiers and new questions. Med Sci Sports Exerc 2007;39:1501. 
3. Trost SG, Mclver KL, Pate RR. Conducting accelerometer-based activity assessments in field-based research. Med Sci Sports Exerc 2005;37: S531-S543.

4. Welk GJ. Principles of design and analyses for the calibration of accelerometry-based activity monitors. Med Sci Sports Exerc 2005;37:S501S511.

5. Yngve A, Nilsson A, Sjostrom M, Ekelund U. Effect of monitor placement and of activity setting on the MTI accelerometer output. Med Sci Sports Exerc 2003;35:320-326.

6. Welk GJ, Blair SN, Wood K, Jones S, Thompson RW. A comparative evaluation of three accelerometry-based physical activity monitors. Med Sci Sports Exerc 2000;32:S489-S497.

7. Nichols JF, Morgan CG, Chabot LE, Sallis JF, Calfas KJ. Assessment of physical activity with the Computer Science and Applications, Inc. accelerometer: laboratory versus field validation. Res Q Exerc Sport 2000;71:36-43.

8. Metcalf BS, Curnow JS, Evans C, Voss LD, Wilkin TJ. Technical reliability of the CSA activity monitor: The EarlyBird Study. Med Sci Sports Exerc 2002;34:1533-1537.

9. Brage S, Brage N, Wedderkopp N, Froberg K. Reliability and validity of the Computer Science and Applications accelerometer in a mechanical setting. Meas Phys Educ Exerc Sci 2003;7:101-119.

10. Brage S, Wedderkopp N, Franks PW, Andersen LB, Froberg K. Reexamination of validity and reliability of the CSA monitor in walking and running. Med Sci Sports Exerc 2003;35:1447-1454.

11. Welk GJ, Schaben JA, Morrow JR. Reliability of accelerometry-based activity monitors: a generalizability study. Med Sci Sports Exerc 2004;36:1637-1645.

12. Rowlands AV, Stone MR, Eston RG. Influence of speed and step frequency during walking and running on motion sensor output. Med Sci Sports Exerc 2007;39:716-727.

13. Trost SG, Ward DS, Moorehead SM, Watson PD, Riner W, Burke JR. Validity of the Computer Science and Applications (CSA) activity monitor in children. Med Sci Sports Exerc 1998;30:629-633.

14. McClain JJ, Sisson SB, Tudor-Locke C. Actigraph accelerometer interinstrument reliability during free-living in adults. Med Sci Sports Exerc 2007;39:1509-1514
15. Nilsson A, Ekelund U, Yngve A, Sjostrom M. Assessing physical activity among children with accelerometers using different time sampling intervals and placements. Pediatr Excer Sci 2002;14:87.

16. Matthews CE, Ainsworth BE, Thompson RW, Bassett DR. Sources of variance in daily physical activity levels as measured by an accelerometer. Med Sci Sports Exerc 2002;34:1376-1381.

17. Bland JM, Altman DG. Measuring agreement in method comparison studies. Stat Methods Med Res 1999;8:135-160.

18. Brage $S$, Wedderkopp $N$, Ekelund $U$, et al. Features of the metabolic syndrome are associated with objectively measured physical activity and fitness in Danish children: The European Youth Heart Study (EYHS). Diabetes Care 2004;27:2141-2148.

19. Bohlmann IM, Mackinnon S, Kruger S, et al. Is the International Physical Activity Questionnaire (IPAQ) valid and reliable in the South African population? Med Sci Sports Exerc 2001;33:S119.

20. Cook I, Lambert EV. Validity and reliability of the International Physical Activity Questionnaire in Northern Sotho-speaking Africans. JEMDSA 2002;7:36.

21. Craig CL, Marshall AL, Sjostrom M, et al. International Physical Activity Questionnaire: 12-country reliability and validity. Med Sci Sports Exerc 2003;35:1381-1395.

22. Welk GJ. Use of accelerometry-based activity monitors to assess physical activity. In: Welk GJ, ed. Physical Activity Assessments for Health-related Research. Champaign, IL: Human Kinetics; 2002. p.125-141.

23. Hopkins WG. Measures of reliability in sports medicine and science. Sports Med 2000;30:1-15.

24. Cohen J. Statistical power analysis for the behavioral sciences. 2nd ed. New York: Academic; 1988.

25. McGraw KO, Wong SP. Forming inferences about some intraclass correlation coefficients. Psychol Methods 1996;1:30-46.

26. Pate RR, Pratt M, Blair SN, et al. Physical activity and public health. A recommendation from the Centers for Disease Control and Prevention and the American College of Sports Medicine. JAMA 1995;273:402-407.

27. Cook I, Lambert EV. The sources of variance and reliability of objectively monitored physical activity in rural and urban Northern Sotho-speaking Africans. S Afr J Sports Med 2008;20:21-27. 